\title{
REVIEW
}

\section{Amyloid Imaging Using High-Field Magnetic Resonance}

\author{
Tomone Amatsubo ${ }^{1,2}$, Daijiro Yanagisawa ${ }^{1,2}$, Shigehiro Morikawa ${ }^{3}$, Hiroyasu Taguchi ${ }^{1}$, \\ and Ikuo Tooyama ${ }^{1 *}$ \\ ${ }^{1}$ Molecular Neuroscience Research Center and ${ }^{3}$ Department of Fundamental Nursing, \\ Shiga University of Medical Science \\ Seta Tsukinowa-cho, Otsu 520-2192, Japan \\ ${ }^{2}$ Japan Science and Technology Agency (JST) Innovation Satellite, Shiga, Japan
}

(Received March 8, 2010; Accepted March 24, 2010)

\begin{abstract}
The formation of senile plaques followed by deposition of amyloid $\beta$ peptides $(\mathrm{A} \beta)$ are the earliest pathological changes of Alzheimer's disease (AD); thus, detection of the plaques remains the most important early diagnostic indicator of AD. Amyloid imaging is a noninvasive technique for visualizing senile plaques in the brains of patients with Alzheimer's using positron emission tomography (PET) or magnetic resonance (MR) imaging. Several types of probes have been developed for PET, but few ligands have been developed specifically for MR imaging detection of amyloid plaques. This review presents recent advances in amyloid imaging using MR imaging and includes our studies.
\end{abstract}

Keywords: Alzheimer's disease, amyloid imaging, cell tracking, contrast agents, ${ }^{19}$ F-MRI

\section{Introduction}

Alzheimer's disease (AD) is a cognitive impairment disorder characterized by cerebral atrophy, deposits of amyloid $\beta$ peptide $(\mathrm{A} \beta)$, neurofibrillary tangles, and neuronal cell loss. According to the amyloid cascade hypothesis for $\mathrm{AD}$, formation of senile plaques followed by the deposition of $\mathrm{A} \beta$ are the earliest pathological changes of AD. ${ }^{1,2}$ Detection of senile plaques is therefore the most important indicator for early diagnosis of AD.

Amyloid imaging is a noninvasive technique that uses positron emission tomography (PET) or magnetic resonance (MR) imaging to visualize $\mathrm{A} \beta$ plaques in the brain. With the increasing incidence of $\mathrm{AD}$ as the average age of many populations increases, amyloid imaging has attracted increasing attention for the early diagnosis of AD. Representative ligands for PET, such as N-methyl- $\left[{ }^{11} \mathrm{C}\right] 2-$ (4-mmethylaminophenyl)-6-hydroxybenzothiazole (PIB) ${ }^{3}$ and 2-[2-(2-dimethylaminothiazol-5-yl) ethenyl]-6-[2-(fluoro)ethoxy] benzoxazole (BF-227), ${ }^{4}$ have been used in clinical research, but no clinical studies of amyloid imaging using MR imaging have been conducted. The likely bottleneck is the lower sensitivity of MR imaging than PET. Many re-

\footnotetext{
*Corresponding author, Phone: +81-77-548-2328, Fax: + 81-
} 77-548-2402, E-mail: kinchan@belle.shiga-med.ac.jp searchers are thus trying to develop novel contrast agents (Table) and pulse sequences ${ }^{5}$ to increase the sensitivity of amyloid detection. We review the trend in amyloid imaging using MR imaging and include results from our studies.

\section{Amyloid Imaging without Contrast Agents}

Senile plaques in the brains of patients with $\mathrm{AD}$ contain iron and iron-binding proteins, such as lactotransferrin and melanotransferrin, ${ }^{6,7}$ and so appear as areas of low density by MR imaging using $\mathrm{T}_{2}{ }^{*}$-weighted gradient echo ${ }^{8}$ and $\mathrm{T}_{2}$-weighted spin echo sequences ${ }^{9,10}$ at high magnetic fields. However, the many hours to obtain a complete image of the senile plaques preclude such studies for in vivo imaging. Amyloid imaging without contrast agents is the ideal alternative, although additional research is needed before applying this approach in human patients. Recently, Chamberlain and colleagues ${ }^{5}$ reported on amyloid plaque contrast generated by a novel imaging method called susceptibility-weighted imaging (SWI). Their ex vivo study presented important observations for the future of in vivo MR imaging of amyloid.

\section{Amyloid Imaging with Contrast Agents}

The essential characteristics of contrast agents 
for amyloid imaging are binding affinity, capacity to pass through the blood-brain barrier (BBB), nontoxicity, and strong signal intensity in the brain. In general, amyloid-binding compound de-

Table. Reports on amyloid imaging with and without contrast agents using magnetic resonance (MR) imaging

\begin{tabular}{lcc}
\hline \multicolumn{1}{c}{ Contrast agent } & $\begin{array}{c}\text { Magnetic } \\
\text { flux density }\end{array}$ & Reference \\
\hline Without & 9.4 tesla (T) & $5,8-10$ \\
Gadolinium $(G d)$-label & & \\
$\quad$ Gd-DTPA-K6A $\beta 1-30$ & $7 \mathrm{~T}$ & 11 \\
Gd-DTPA-A $\beta 1-40$ & $7 \mathrm{~T}$ & 12,13 \\
PUT-Gd-A $\beta 1-40$ & $7 \mathrm{~T}$ & 13 \\
Gd-A $\beta 1-40$ & $7 \mathrm{~T}$ & 13 \\
Gd-DOTA-pF $\left(\mathrm{ab}^{\prime}\right)_{2} 4.1$ & $9.4 \mathrm{~T}$ & 14 \\
${ }^{19}$ F-labels & & 15 \\
$\quad \begin{array}{l}\text { FSB } \\
\text { Polyfluorinated }\end{array}$ & $9.4 \mathrm{~T}$ & 16 \\
bis-styrylbenzene & - & 17 \\
$\begin{array}{l}\text { TFMB } \\
\text { Other labels }\end{array}$ & $7 \mathrm{~T}$ & 12 \\
$\quad \begin{array}{l}\text { MION-A } \beta 1-40 \\
\text { Resovist-labeled } \\
\text { microglia }\end{array}$ & $7 \mathrm{~T}$ & $18-20$ \\
\hline
\end{tabular}

Abbreviations and acronyms

FSB: (E,E)-1 - fluoro-2,5-bis(3 - hydroxycarbonyl-4-hydroxy)styrylbenzene, Gd-DOTA; gadolinium-tetraazacyclododecanetetraacetic acid, Gd-DTPA: gadopentetate dimeglumine, MION: monocrystalline iron oxide nanoparticles PUT: putrescine, TFMB: trifluoromethoxy-benzylate. rivatives and paramagnetic-labeled compounds are used.

\section{Gadolinum-labeled contrast agents}

Gadolinium $(\mathrm{Gd})$ is a typical contrast agent used for MR imaging. Gd-labeled $\mathrm{A} \beta$ peptide ${ }^{11-13}$ and $\mathrm{A} \beta$ antibody ${ }^{14}$ have been injected into transgenic mice with $\mathrm{AD}$ to detect amyloid plaques in the mouse brain. Using high magnetic field MR systems, senile plaques in the mouse brain are visualized with $T_{1}$-weighted and $T_{2}$-weighted $M R$ images as the high and low signal intensities, respectively. The above results suggested that amyloid plaque might also be detected using other magnetically labeled $\mathrm{A} \beta$ peptide and $\mathrm{A} \beta$ antibody, such as monocrystalline iron oxide nanoparticle (MION)-A $\beta 1$ $40 .{ }^{12}$

\section{2. ${ }^{19} \mathrm{~F}$-labeled contrast agents}

The advantage of ${ }^{19} \mathrm{~F}-\mathrm{MR}$ imaging is the high sensitivity of the ${ }^{19} \mathrm{~F}$ to MR ratio $\left({ }^{1} \mathrm{H}:{ }^{19} \mathrm{~F}=1: 0.83\right)$, high natural abundance ratio (100\%), and absence of endogenous ${ }^{19} \mathrm{~F}$. However, disadvantages to amyloid imaging by ${ }^{19} \mathrm{~F}-\mathrm{MR}$ imaging include the low concentration accumulated in the body and the lack of clarity regarding chemical properties of probes that improve amyloid detection.

(E,E)-1-fluoro-2,5-bis(3-hydroxycarbonyl-4-hydroxy)styrylbenzene (FSB) (Fig. 1a), ${ }^{15}$ bis-styrylbenzenes (Fig. 1b, c), ${ }^{16}$ and trifluoromethoxy-benzylated (TFMB) compounds (Fig. 1d, e) ${ }^{17}$ have been reported as amyloid imaging ligands for ${ }^{19} \mathrm{~F}$ MR imaging. FSB allowed the detection of $\mathrm{A} \beta$

a

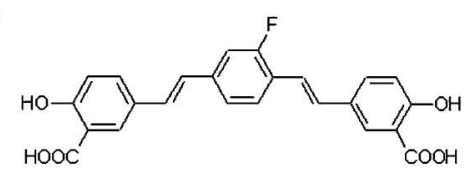

FSB

b

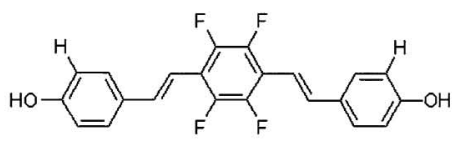

bis-styrylbenzene 8
C

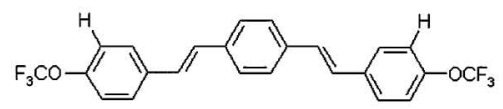

bis-styrylbenzene 11<smiles>COc1cccc(CNCCOCCOc2ccc3nc(/C=C/c4ccc(N(C)C)cc4)oc3c2)c1</smiles>

TFMB-2Et e<smiles>COc1cccc(CNCCOCCOCCOc2ccc3nc(CCc4ccc(N(C)C)cc4)oc3c2)c1</smiles>

TFMB-3Et

Fig. 1. Structure of amyloid imaging ligands for ${ }^{19} \mathrm{~F}$-magnetic resonance imaging 
plaques in transgenic mice with amyloid precursor protein (APP) using 9.4-tesla MR imaging, ${ }^{15}$ but we could not detect the ${ }^{19} \mathrm{~F}$-signal of injected FSB $(200 \mu \mathrm{g} / \mathrm{head})$ in mouse brain at $7 \mathrm{~T}$. We therefore investigated developing novel ${ }^{19} \mathrm{~F}$-labeled amyloidimaging ligands for MR imaging and improved instrumentation.

First, we synthesized 2-(4'-dimethylaminostyryl)6-\{2-[(3'-trifluoromethoxy)benzylamino] ethoxyethoxy benzoxazole (TFMB-2Et) and 2-(4'-dimethylaminostyryl)-6-\{2-[(3'-trifluoromethoxy) benzylamino]ethoxyethoxyethoxy\}benzoxazole (TFMB$3 \mathrm{Et}$ ), both of which have stronger ${ }^{19} \mathrm{~F}$-signals than FSB. We attempted to localize TFMB-2Et and TFMB-3Et in mouse muscle and brain using 7T MR imaging, but detecting ${ }^{19} \mathrm{~F}$-signal in mouse brain was difficult. ${ }^{17}$ Ex vivo MR imaging and in vitro nuclear magnetic resonance (NMR) analyses showed the NMR signals of ${ }^{19} \mathrm{~F}$ to be very sensitive to the tissue environment and that brain tissues inhibit amyloid detection in ${ }^{19} \mathrm{~F}-\mathrm{MR}$ imaging by affecting the ${ }^{19} \mathrm{~F}-\mathrm{NMR}$ signals. ${ }^{17}$ Comparing the $\log P$ values and the intensities of ${ }^{19} \mathrm{~F}$-NMR signals in brain homogenates using several compounds with a trifluoro-base revealed that hydrophilic chemicals (low $\log P$ ) have relatively strong ${ }^{19} \mathrm{~F}$ NMR signals. Therefore, effective MR imaging of amyloid in the brain requires optimizing probe hydrophilicity while retaining good brain permeability of the probe.

Second, we optimized the radiofrequency (RF) coil because we detected an unexpectedly high ${ }^{19} \mathrm{~F}$ signal with our home-built, solenoid-type volume coil designed to detect the ${ }^{19} \mathrm{~F}$ signal of TFMB-2Et (Fig. 2A). The ${ }^{19} \mathrm{~F}$ chemical shift image of a TFMB2Et tube in 3 orthogonal planes with a larger field of view showed ${ }^{19} \mathrm{~F}$-signal of the TFMB-2Et (Fig. 3D-I, arrow) and additional high signal from the capacitors (Fig. 3 D-I, arrowhead). We successfully removed the noise by replacing the existing capacitor with glass parts (Fig. 2B). Attention should also be paid to instrument noise in detecting weak ${ }^{19} \mathrm{~F}$ signals.

\section{Amyloid Imaging by Cell Tracking}

Because microglia accumulate around senile plaques and remove $\mathrm{A} \beta$ deposits in the brain, ${ }^{18}$ we could track microglia around senile plaques by MR imaging. Because native cells are not detected by MR imaging, cells must be labeled with a contrast agent for MR tracking. The most popular such agents are superparamagnetic iron oxides (SPIOs). $\mathrm{We}^{19}$ and others ${ }^{20}$ have developed a highly efficient labeling method using a nonviral vector system, the hemagglutinating virus of Japan (HVJ; Sendai virus) envelope (HVJ-E) vector. The high effectiveness of HVJ-E vector labeling allowed us to detect a few cells at each spot in the mouse brain using MR imaging. ${ }^{21}$

We then injected $\beta$-amyloid peptide $\mathrm{A} \beta 1-42$ (A $\beta 42)$ into the left hippocampus and saline into the right hippocampus of rats and labeled microglia with a SPIO (Resovist, Bayer, Osaka, Japan) administered via the carotid artery or lateral ventricle. MR imaging revealed clear signal changes attributable to Resovist-containing microglia accumulated in the areas injected with $\mathrm{A} \beta 42 .{ }^{22,23}$ Because microglia accelerate the removal of $\mathrm{A} \beta$, this technique might also be applicable to amyloid imaging and cell therapy for AD.

\section{Conclusion}

Although many researchers have addressed the development of novel contrast agents and pulse sequence to increase the sensitivity of amyloid detection, many issues still exist in using amyloid imaging for MR imaging in humans. However, such application of MR imaging is very useful in terms of safety, cost, and apparatus penetration rate and is expected to progress in the future.

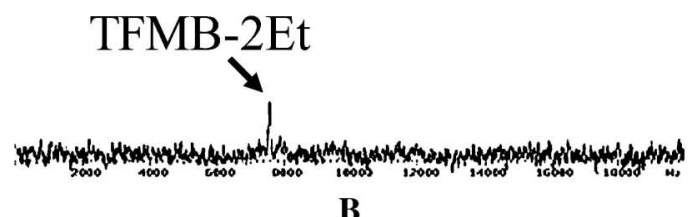

Fig. 2. ${ }^{19}$ F-single pulse spectra of 2-(4'-dimethylaminostyryl)-6-\{2-[(3'-trifluoromethoxy) benzylamino]ethoxyethoxy benzoxazole (TFMB-2Et) corrected by home-made coil (A) and new homemade coil with the glass parts of the capacitors replaced $(\mathbf{B})$. 


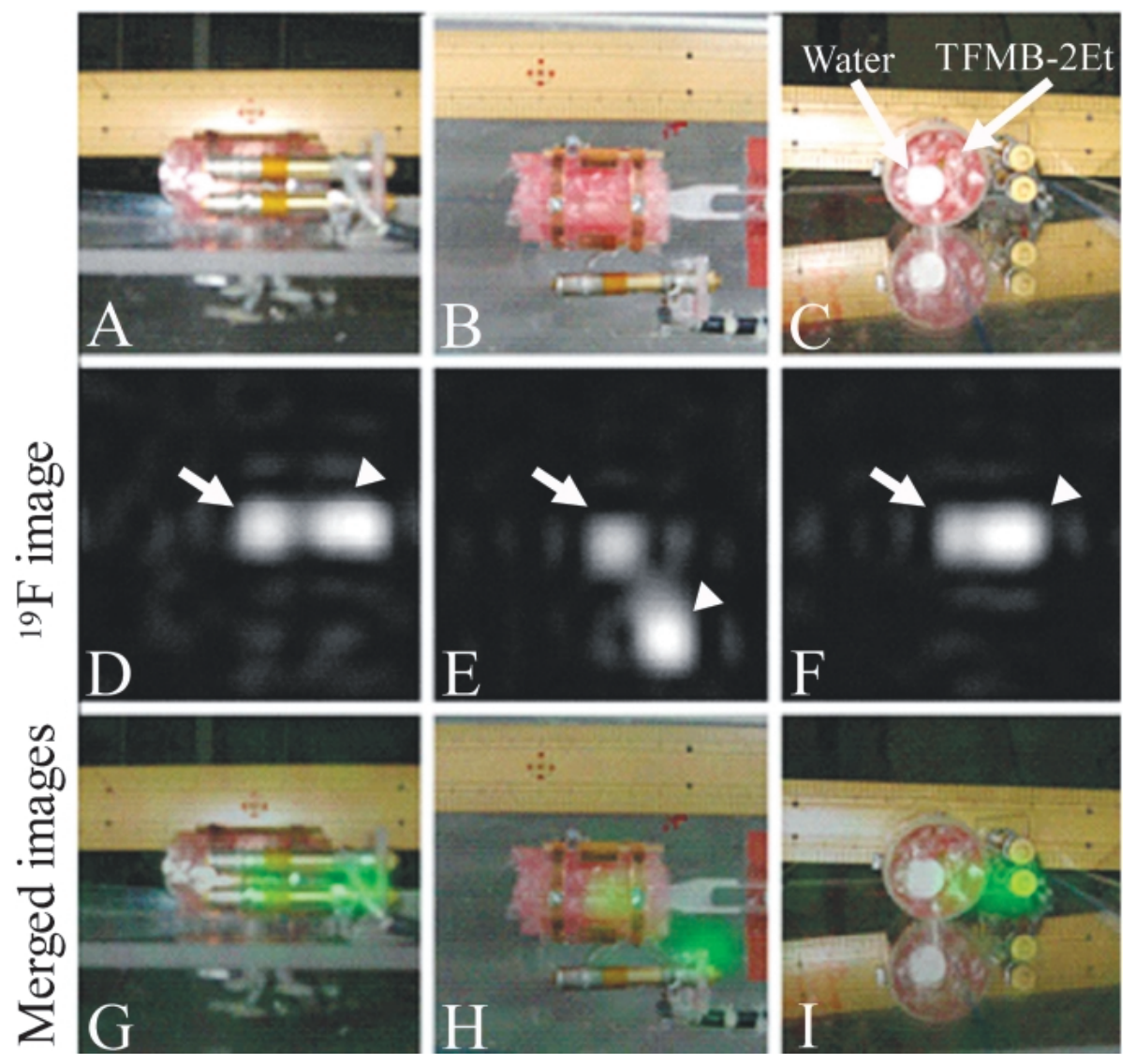

Fig. 3. ${ }^{19}$ F-chemical shift imaging of 2-(4'-dimethylaminostyryl)-6- $\left\{2-\left[\left(3^{\prime}-\right.\right.\right.$ trifluoromethoxy) benzylamino]ethoxyethoxy benzoxazole (TFMB-2Et) (arrow) and noise (arrowhead) in the 3 orthogonal planes with bigger field of view A, B: View showing 3 orthogonal planes of a home-built, solenoid-type volume coil. C-E: ${ }^{19}$ F-nuclear magnetic resonance (NMR) signals obtained by chemical shift imaging. F-H: Merged images.

\section{Acknowledgments}

This study was supported by the Japan Science and Technology Agency (JST) Practical Application Research Program and Grants-in Aid for Scientific Research (No. 1930058).

\section{References}

1. Hardy JA, Higgins GA. Alzheimer's disease: the amyloid cascade hypothesis. Science 1992; 256: 184-185.

2. Hardy JA, Selkoe DJ. The amyloid hypothesis of Alzheimer's disease: progress and problems on the road to therapeutics. Science 2002; 297:353-356.

3. Klunk WE, Engler H, Nordberg A, et al. Imaging brain amyloid in Alzheimer's disease with Pittsburgh Compound-B. Ann Neurol 2004; 55:306319.

4. Kudo Y, Okamura N, Furumoto S, et al. 2-(2-[2Dimethylaminothiazol-5-yl]ethenyl)-6-(2-[fluoro] ethoxy)benzoxazole: a novel PET agent for in vivo detection of dense amyloid plaques in Alzheimer's disease patients. J Nucl Med 2007; 48:553-561.

5. Chamberlain R, Reyes D, Curran GL, et al. Comparison of amyloid plaque contrast generated by $\mathrm{T}_{2}$-weighted, $\mathrm{T}_{2}{ }^{*}$-weighted, and susceptibilityweighted imaging methods in transgenic mouse models of Alzheimer's disease. Magn Reson Med 2009; 61:1158-1164.

6. Leveugle B, Spik G, Perl DP, Bouras C, Fillit HM, Hof PR. The iron-binding protein lactotransferrin is present in pathologic lesions in a variety of neurodegenerative disorders: a comparative immunohistochemical analysis. Brain Res 1994; 650:20-31.

7. Connor JR, Menzies SL, St. Martin SM, Mufson EJ. A histochemical study of iron, transferrin, and ferritin in Alzheimer's diseased brains. J Neurosci Res 1992; 31:75-83.

8. Jack CR Jr, Garwood M, Wengenack TM, et al. In vivo visualization of Alzheimer's amyloid plaques by magnetic resonance imaging in transgenic mice without a contrast agent. Magn Reson Med 2004; 
52:1263-1271.

9. Jack CR Jr, Wengenack TM, Reyes DA, et al. In vivo magnetic resonance microimaging of individual amyloid plaques in Alzheimer's transgenic mice. J Neurosci 2005; 25:10041-10048.

10. Zhang J, Yarowsky P, Gordon MN, et al. Detection of amyloid plaques in mouse models of Alzheimer's disease by magnetic resonance imaging. Magn Reson Med 2004; 51:452-457.

11. Sigurdsson EM, Wadghiri YZ, Mosconi L, et al. A non-toxic ligand for voxel-based MRI analysis of plaques in AD transgenic mice. Neurobiol Aging 2007; 29:836-847.

12. Wadghiri YZ, Sigurdsson EM, Sadowski M, et al. Detection of Alzheimer's amyloid in transgenic mice using magnetic resonance microimaging. Magn Reson Med 2003; 50:293-302.

13. Poduslo JF, Wengenack TM, Curran GL, et al. Molecular targeting of Alzheimer's amyloid plaques for contrast-enhanced magnetic resonance imaging. Neurobiol Dis 2002; 11:315-329.

14. Ramakrishnan M, Wengenack TM, Kandimalla $\mathrm{KK}$, et al. Selective contrast enhancement of individual Alzheimer's disease amyloid plaques using a polyamine and Gd-DOTA conjugated antibody fragment against fibrillar $\mathrm{A} \beta_{42}$ for magnetic resonance molecular imaging. Pharm Res 2008; 25:1861-1872.

15. Higuchi M, Iwata N, Matsuba Y, Sato K, Sasamoto K, Saido CT. ${ }^{19} \mathrm{~F}$ and ${ }^{1} \mathrm{H}$ MRI detection of amyloid beta plaques in vivo. Nat Neurosci 2005; 8:527-533.

16. Flaherty DP, Walsh SM, Kiyota T, Dong Y, Ikezu T, Vennerstrom JL. Polyfluorinated bis-styrylben- zene $\beta$-amyloid plaque binding ligands. J Med Chem 2007; 50:4986-4992.

17. Amatsubo $\mathrm{T}$, Morikawa $\mathrm{S}$, Inubushi $\mathrm{T}$, et al. Trifluoromethoxy-benzylated ligands improve amyloid detection in the brain using ${ }^{19} \mathrm{~F}$ magnetic resonance imaging. Neurosci Res 2009; 63:76-81.

18. Akiyama H, Barger S, Barnum S, et al. Inflammation and Alzheimer's disease. Neurobiol Aging 2000; $21: 383-421$.

19. Toyoda K, Tooyama I, Kato M, et al. Effective magnetic labeling of transplanted cells with HVJ-E for magnetic resonance imaging. Neuroreport 2004; 15:589-593.

20. Miyoshi S, Flexman JA, Cross DJ, et al. Transfection of neuroprogenitor cells with iron nanoparticles for magnetic resonance imaging tracking: cell viability, differentiation, and intracellular localization. Mol Imaging Biol 2005; 7:286-295.

21. Song Y, Morikawa S, Morita M, et al. Magnetic resonance imaging using hemagglutinating virus of Japan-envelope vector successfully detects localization of intra-cardially administered microglia in normal mouse brain. Neurosci Lett 2006; 395:4245.

22. Takata T, Kitamura Y, Yanagisawa D, et al. Microglia transplantation increases amyloid- $\beta$ clearance in Alzheimer model rats. FEBS Lett 2007; 581:475-478.

23. Song Y, Morikawa S, Morita M, et al. Comparison of MR images and histochemical localization of intra-arterially administered microglia surrounding $\beta$-amyloid deposits in the rat brain. Histol Histopathol 2006; 21:705-711. 